\title{
Toxicity of abdominal fat
}

\author{
Shawn Mathew ${ }^{1}$, Constantine E. Kosmas ${ }^{2}$, Robert R. Siegel ${ }^{3}$, Timothy J. Vittorio ${ }^{4^{*}}$ \\ ${ }^{1}$ New York Institute of Technology, Old Westbury, New York, USA \\ ${ }^{2}$ Zena and Michael A. Wiener Cardiovascular Institute, Mount Sinai School of Medicine, New York, USA \\ ${ }^{3}$ Division of Cardiology, Albert Einstein College of Medicine, Bronx Municipal Hospital Center, Bronx, USA \\ ${ }^{4}$ St. Francis Hospital-The Heart Center ${ }^{\circledR}$, Division of Cardiology, Center for Advanced Cardiac Therapeutics, Roslyn, USA \\ *Corresponding Author: t_vittorio@hotmail.com
}

Received 20 June 2013; revised 20 July 2013; accepted 10 August 2013

Copyright (C) 2013 Shawn Mathew et al. This is an open access article distributed under the Creative Commons Attribution License, which permits unrestricted use, distribution, and reproduction in any medium, provided the original work is properly cited.

\begin{abstract}
Abdominal fat or truncal obesity consists of both subcutaneous and visceral fat. It has been found that visceral fat is more metabolically active than subcutaneous fat. The low-level inflammation linked with abdominal fat is associated with insulin resistance and with increases in the release of inflammatory adipokines and cytokines. As a result of these changes, abdominal fat can cause a variety of health conditions. In this review, we focus on the adverse effects of abdominal fat on the body and how it can lead to the development of cardiovascular disease, hypertension, type 2 diabetes mellitus, hyperlipidemia, stroke and cancer. Additionally, we discuss how abdominal fat can be reduced as a result from correction of hormonal deficiencies.
\end{abstract}

Keywords: Adipokines; Cytokines; Fat; Hormonal; Inflammation; Obesity

\section{INTRODUCTION}

The obesity pandemic plagues many lives and poses a tremendous health risk. Obesity can be classified as either android or gynoid obesity [1]. Android obesity occurs when fat is deposited in the abdominal (truncal) region. Gynoid obesity occurs when fat is deposited in the buttocks and thighs (hips). Android obesity is more of a health concern than gynoid obesity. There are several ways in which we can measure the degree of obesity in an individual. By measuring the body weight and height, one can calculate the body mass index (BMI) specific to that person. An adult with a BMI of $\geq 30.0 \mathrm{~kg} / \mathrm{m}^{2}$ or $\geq 40$ $\mathrm{kg} / \mathrm{m}^{2}$ is classified as obese or morbidly obese, respectively [2]. By measuring waist circumference, we can determine if an individual possesses a health risk that is associated with body weight. Women with a waist circumference $\geq 80 \mathrm{~cm}$ and men with a waist circumference $\geq 94 \mathrm{~cm}$ are at an increased health risk [2].

Generally, obesity is caused when energy consumption exceeds the amount of energy expended. In order to compensate for the extra energy, adipose tissue is utilized in energy storage, and when there is a large demand of lipid storage, adipocyte hypertrophy occurs [2]. In adipocyte hypertrophy, fat cells increase their volume so that they can withstand the large lipid storage. Thus, this results in weight gain in adults. Adipose tissue can be categorized into white and brown adipose tissue and brown adipose tissue. Adipose tissue mainly consists of white adipose tissue which is used in energy storage, whereas brown adipose tissue is used as a homeostatic mechanism [3]. In the abdomen, fat can be stored as either visceral fat or subcutaneous fat. When normal subcutaneous adipose tissue storage sites are full, and the body needs to store excess triglycerides, the excess triglycerides are stored in abnormal subcutaneous adipose tissue storage sites. Abnormal subcutaneous adipose storage sites have a decreased ability to store fatty acids and can also cause an abundant release of free fatty acids (FFAs) [4]. It has been determined that upper body subcutaneous fat is more lipolytically active than lower body fat. However, visceral fat is more lipolytically active than subcutaneous fat. Thus, visceral fat is more of a health threat than subcutaneous fat. It has been found that higher levels of abdominal subcutaneous adipose tissue and abdominal visceral adipose tissue have been shown to increase the risk of cardiometabolic conditions [5]. Effective methods of assessing abdominal fat are dual-energy X-ray absorptiometry (DEXA) and anthropometric measures, which are instrumental in the calculation of visceral fat [6].

\section{PATHOPHYSIOLOGY}

Abdominal fat causes a variety of changes throughout 
the body, which are elicited through triggering of the immune system. This can be categorized as low-level chronic inflammation. Chronic inflammation can lead to insulin resistance, which in turn causes increases in the levels of adipokines, such as serum Amyloid-A and resistin, as well as in the levels of inflammatory cytokines, such as tumor necrosis factor (TNF- $\alpha$ ), interleukin-6 (IL6) and monocyte chemotactic protein (MCP-1) [7]. Serum Amyloid-A is an adipokine that directly influences the cells of the immune system to cause an increase in the production of inflammatory cytokines [7]. Resistin is an inflammatory cytokine that can increase cytokine production in macrophages. Since resistin production is controlled by the inflammatory cytokines, the increase in cytokine production amplifies the response. Thus, the system shifts away from equilibrium, as a result of positive feedback.

\subsection{Cardiovascular Disease}

The occurrence of chronic inflammation is associated with an increased risk of cardiovascular disease [3]. Low-level chronic inflammation disrupts cardiovascular function and it can even accelerate atherosclerosis [7]. According to the INSPIRE ME IAA Study, increased abdominal circumference associated with visceral fat is strongly associated with cardiovascular disease [8]. Epicardial adipose tissue is visceral fat deposited in the chest cavity, which covers the coronary arteries and muscular tissue of the heart. Epicardial adipose tissue is linked with high-risk plaques and atherosclerosis [9]. It has been found that individuals who suffer from coronary artery disease (CAD) have increased epicardial adipose tissue thickness and volume, as compared to individuals devoid of CAD [9].

\subsection{Type 2 Diabetes Mellitus}

Hyperinsulinemia and insulin resistance are both associated with an increased risk of type 2 diabetes mellitus [10]. In the body, visceral fat is a source of lipids. These lipids are transported to the liver via the hepatic portal vein. The accumulation of these lipids interferes with insulin signaling in liver cells [6]. This occurs because excess FFAs can alter blood glucose levels through several mechanisms, including excess of acetylcoenzyme A, as well as a reduction of nicotinamide adenine dinucleotide, ATP and citrate [4]. Increased levels of adipokines and cytokines are also linked to visceral fat. The latter increased levels of cytokines can deter insulin receptor kinase activity, thus disrupting insulin signaling by inhibiting the transmission of chemical responses within cells [7]. In addition, obesity causes a decrease in insulin sensitivity of the liver and thereby leading to insulin resistance [10]. Thus, the liver extracts less insulin than usual. Furthermore, visceral fat causes an increase in the amount of secreted insulin which enters the blood. The decrease in extracted insulin by the liver and increase in the amount of secreted insulin entering the blood can cause hyperinsulinemia.

\subsection{Hyperlipidemia}

The metabolism of lipoproteins is controlled by hepatic triglyceride lipase (HTGL) [11]. When the concentration of post-hepatic HTGL is high, then the serum concentration of high-density lipoprotein (HDL) cholesterol is low. In visceral adipose tissue there is high HTGL activity. Thus, visceral fat causes low levels of HDL cholesterol (also referred to as "good cholesterol"). Visceral fat also contains high levels of triglycerides. The abundance of triglycerides is linked with high concentrations of very-low-density lipoprotein (VLDL) as well as lowdensity lipoprotein (LDL). Thus, visceral fat can cause increased concentrations of VLDL and LDL in the blood.

\subsection{Hypertension}

Hypertension is a condition in which there is elevated systemic blood pressure. Adiponectin is an adipokine that is secreted by adipocytes; however, in obesity, there are decreased levels of adiponectin [12]. Adiponectin is a vasodilator and it helps to regulate blood pressure. In obese individuals, subcutaneous adipose tissue expresses a greater amount of adiponectin than in visceral adipose tissue. Thus, excess visceral adipose tissue is a factor which can lead to the development of systemic hypertension through low-level chronic inflammation and decreased levels of adiponectin.

\subsection{Stroke}

Obesity is associated with an environment that promotes inflammation and thrombosis [13]. This type of environment can lead to the development of stroke. Specifically, it has been shown that an increase in abdominal obesity leads to an increased risk of cerebral thrombosis. In the human body, the carotid arteries provide the majority of blood to the brain. The Multicultural Community Health Assessment Trial (M-CHAT) Study demonstrated that increased abdominal girth, in particular, visceral adipose tissue is associated with the development of carotid atherosclerosis [14].

\subsection{Cancer}

As we previously discussed, obesity is associated with low-level chronic inflammation. In this environment, macrophages are triggered and these macrophages are able to enter tumors. When the macrophages enter tumors, they produce cytokines, prostaglandins and angiogenic 
factors, which in turn aid tumor growth [15]. Plasminogen activator inhibitor-1 (PAI-1) is a protein that is made in visceral white adipose tissue. Increased PAI-1 levels can lead to the formation of new blood vessels, which can promote tumor cell growth and cause the tumor cells to metastasize [15].

\section{DISCUSSION}

\subsection{How to Reduce Visceral Fat}

Despite a heritability of $48 \%$ - $57 \%$ for visceral fat [6], there are several methods to maintain low-levels of abdominal fat. Exercise is a critical way of reducing abdominal fat. Intense physical activity can improve overall health [2]. It is recommended that individuals partake in at least 30 minutes of high-intensity physical activity daily. Additionally, resistance strength training is important, because it helps maintain muscle mass when an individual is trying to lose weight via diet [2]. Moreover, a well-rounded low-glycemic diet is effective in combating obesity. Effective diets are diets that reduce the total energy intake and result in the most weight loss. Converting from a diet that includes refined grain foods to a diet that includes whole grain foods has shown to decrease visceral fat [16]. It has been found that two drugs are effective in reducing belly fat: Sibutramine and Orlistat [2]. Sibutramine prevents the reuptake of certain neurotransmitters, which results in decreased food intake. On the other hand, Orlistat decreases the absorption of triglycerides in the small intestine. In severe cases of obesity, gastric bypass surgery can be performed. In this type of surgery, the stomach is stapled and this creates a small pouch. This reduces food intake, and since a portion of the stomach and a portion of the small intestine are bypassed, certain nutrients cannot be absorbed.

\subsection{Hormonal Deficiencies (HGH, Testosterone and DHEA)}

Hormonal deficiencies can also be the root of abdominal fat. Adults with acquired human growth hormone $(\mathrm{HGH})$ deficiency have increased visceral adipose tissue [17]. As the extent of obesity increases in adults, growth hormone secretion decreases within the body. Even in nonobese individuals, abdominal fat has shown to decrease growth hormone secretion [17].

In men, testosterone is vital in order to decrease fat and to preserve muscle. Therefore, testosterone deficiency can lead to obesity. In addition, low levels of testosterone can lead to the development of metabolic syndrome and type 2 diabetes mellitus [18]. This occurs because low levels of testosterone lead to increased adipokine release by the adipocytes, which in turn can lead to the chronic low-level inflammatory state associated with obesity. When testosterone is restored to an ade- quate level, it reduces triglyceride storage and lipoprotein lipase (LPL) activity [18].

Dehydroepiandrosterone (DHEA) is a hormone secreted by the adrenal gland. Decreased levels of DHEA sulfate are associated with obesity. It has also been found that DHEA sulfate stimulates adiponectin gene expression in visceral fat [19]. Therefore, DHEA sulfate deficiency can lead to decreased levels of adiponectin, and subsequently abdominal obesity [19].

\section{CONCLUSION}

The increase in abdominal fat is associated with the risk of developing cardiovascular disease, type 2 diabetes mellitus, hypertension, stroke and certain cancers. Abdominal fat contains both visceral and subcutaneous adipose tissue, but visceral adipose tissue poses more of a health threat. It has been found that exercise, diet, prescribed drugs and surgery are viable options which can effectively reduce abdominal fat. However, hormonal deficiencies of $\mathrm{HGH}$, testosterone and DHEA can lead to the rise of abdominal fat. All in all, it is important to acknowledge the hazardous effects of abdominal fat and to know how to effectively manage it.

\section{REFERENCES}

[1] Ribisl, P.M. (2004) Clinical applications: Toxic "waist" dump: Our abdominal visceral fat. ACSM's Health and Fitness Journal, 8, 22-25. doi:10.1097/00135124-200407000-00007

[2] Racette, S.B., Deusinger, S.S. and Deusinger, R.H. (2003) Obesity: Overview of prevalence, etiology, and treatment. Physical Therapy, 83, 276-288.

[3] Fantuzzi, G. (2005) Adipose tissue, adipokines, and inflammation. Journal of Allergy and Clinical Immunology, 115, 911-919. doi:10.1016/j.jaci.2005.02.023

[4] Jenson, M.D. (2008) Role of body fat distribution and the metabolic complications of obesity. The Journal of Clinical Endocrinology \& Metabolism, 93, S57-S63. doi:10.1210/jc.2008-1585

[5] Liu, J., Fox, C.S., Hickson, D.A., May, W.D., Hairston, K.G., Carr, J.J. and Taylor, H.A. (2010) Impact of abdominal visceral and subcutaneous adipose tissue on cardiometabolic risk factors: The jackson heart study. The Journal of Clinical Endocrinology \& Metabolism, 95, 5419-5426. doi:10.1210/jc.2010-1378

[6] Direk, K., Cercelia, M., Astle, W., Chowienczyk, P., Spector, T.D., Falchi, M. and Andrew, T. (2013) The relationship between DXA-based and anthropometric measures of visceral fat and morbidity in women. BMC Cardiovascular Disorders, 13, 25. doi:10.1186/1471-2261-13-25

[7] Alam, I., Ng, T.P. and Larbi A. (2012) Does inflammation determine whether obesity is metabolically healthy or unhealthy? The aging perspective. Mediators of Inflammation, 2012, 456456. doi:10.1155/2012/456456 
[8] Smith, J.D., Borel, A.L., Nazare, J.A., Haffner, S.M., Balkau, B., Ross, R., Massien, C., Almèras, N. and Desprès, J.P. (2012) Visceral adipose tissue indicates the severity of cardiometabolic risk in patients with and without type 2 diabetes: Results from the inspire me IAA study. The Journal of Clinical Endocrinology \& Metabolism, 97, 1517-1525. doi:10.1210/jc.2011-2550

[9] Echavarría-Pinto, M., Hernando, L. and Alfonso, F. (2013) From the epicardial adipose tissue to vulnerable coronary plaques. World Journal of Cardiology, 5, 68-74. doi:10.4330/wjc.v5.i4.68

[10] Hsu, I.R., Kim, S.P., Kabir, M. and Bergman, R.N. (2007) Metabolic syndrome, hyperinsulinemia, and cancer. The American Journal of Clinical Nutrition, 86, 867S-871S.

[11] Ebbert, J.O. and Jensen, M.D. (2013) Fat depots, free fatty acids, and dyslipidemia. Nutrients, 5, 498-508. doi:10.3390/nu5020498

[12] Summer, R., Walsh, K. and Medoff, B.D. (2011) Obesity and pulmonary arterial hypertension: Is adiponectin the molecular link between these conditions? Pulmonary Circulation, 1, 440-447. doi:10.4103/2045-8932.93542

[13] Zhang, X., Shu, X.O., Gao, Y.T., Yang, G., Li, H. and Zheng, W. (2009) General and Abdominal adiposity and risk of stroke in Chinese women. Stroke, 40, 1098-1104. doi:10.1161/STROKEAHA.108.539692

[14] Lear, S.A., Humphries, K.H., Kohli, S., Frohlich, J.J., Birmingham, C.L. and Mancini, G.B.J. (2007) Visceral adipose tissue, a potential risk factor for carotid atherosclerosis: Results of the multicultural community health assessment trial (M-CHAT). Stroke, 38, 2422-2429. doi:10.1161/STROKEAHA.107.484113

[15] Hursting, S.D. and Dunlap, S.M. (2012) Obesity, metabolic dysregulation, and cancer: A growing concern and an inflammatory (and microenvironmental) issue. Annals of the New York Academy of Science, 1271, 82-87. doi:10.1111/j.1749-6632.2012.06737.x

[16] Katcher, H.I., Legro, R.S., Kunselman, A.R., Gillies, P.J., Demers, L.M., Bagshaw, D.M. and Kris-Etherton, P.M. (2008) The effects of a whole grain-enriched hypocaloric diet on cardiovascular disease risk factors in mean and women with metabolic syndrome. The American Journal of Clinical Nutrition, 87, 79-90.

[17] Shalet, S.M. (2010) Partial growth hormone deficiency in adults; should we be looking for it? Clinical Endocrinology, 73, 432-435.

[18] Saad, F., Aversa, A., Isidori, A.M. and Gooren, L.J. (2012) Testosterone as potential effective therapy in treatment of obesity in men with testosterone deficiency: A review. Current Diabetes Reviews, 8, 131-143. doi:10.2174/157339912799424573

[19] Hernandez-Morante, J.J., Milagro, F., Gabaldon, J.A., Martinez, J.A., Zamora, S. and Garaulet M. (2006) Effect of DHEA-sulfate on adiponectin gene expression in adipose tissue from different fat depots in morbidly obese humans. European Journal of Endocrinology, 155, 593600. $\underline{\text { doi: } 10.1530 / \text { eje. } 1.02256}$

\author{
ABBREVIATIONS \\ ATP: Adenosine triphosphate \\ BMI: Body mass index \\ CAD: Coronary artery disease \\ DHEA: Dehydroepiandrosterone \\ DXA: Dual-energy X-ray absorptiometry \\ HDL: High-density lipoprotein \\ HGH: Human growth hormone
}

HTGL: Hepatic triglyceride lipase

IL-6: Interleukin-6

LDL: Low-density lipoprotein

LPL: Lipoprotein lipase

MCP-1: Monocyte chemotactic protein

PAI- 1: Plasminogen activator inhibitor-1

TNF- $\alpha$ : Tumor necrosis factor

VLDL: Very-low-density lipoprotein 